\title{
Hydrodynamic Description of Planetary Rings
}

\author{
Frank Spahn* and Jürgen Schmidt \\ Arbeitsgruppe Nichtlineare Dynamik, Universität Potsdam, Am Neuen Palais 10, 14469 Pots- \\ dam
}

Received 24 February 2005, revised 21 April 2005, accepted 18 May 2005

Key words Hydrodynamics, planetary rings, astrophysics, kinetic theory, stability

We demonstrate the suitability of a hydrodynamic description of the dynamics of a dense planetary ring considering as an example the viscous oscillatory instability. For the successful application of hydrodynamics the transport coefficients must be known as functions of surface mass density $\sigma$ and the granular temperature $T$ of the ring material. We arrange scaling laws for these quantities and use parameters determined from particle simulations of a planetary ring. With such a preparation, the theory predicts an oscillatory instability of a dense ring: waves of radial wavelengths $\lambda \approx 100 \mathrm{~m} . .200 \mathrm{~m}$ evolve, driven by the combined effects of viscous momentum flux and Coriolis force. These predictions are confirmed by direct particle simulations: wavelengths of the unstable modes, their growth rates, as well as the properties of the eigenfunctions of the perturbations. This success bears the hope to explain a class of so-called fine-structure in Saturn's rings, which are poorly understood to date. The experiments of the Cassini-spacecraft in orbit around Saturn offer the unique chance to identify the predicted density features by analyzing the imaging and occultation data.

(c) 2006 WILEY-VCH Verlag GmbH \& Co. KGaA, Weinheim

\section{Introduction}

Since the advent of space exploration in the second half of the 20th century the knowledge about and interest in planetary rings surrounding the giant planets of our Solar system increased dramatically. Before this time Saturn's rings had been considered as a beautiful but unique whim of nature. When James Elliot and his team observed a stellar occultation of Uranus in 1978 they found an unexpected significant reduction of the starlight intensity definitely away from the planet itself: The Uranian rings were detected. The exciting discoveries of the rings of Jupiter and Neptune by the Voyager space-probe then marked the beginning of a new branch of planetary sciences - the systematic study of planetary rings. It became clear that rings are common to giant planets just like their pronounced satellite systems. The planetary rings we observe in the Solar system have many physical properties in common with accretion disks around stars, proto-planetary disks, and with galactic disks.

Among the four ring systems Saturn's rings are by far the largest, most diverse and impressive ones. They show most of the features observed in the ring systems of the other giant

\footnotetext{
* Corresponding author; email: frank@agnld.uni-potsdam.de, Phone: +49 (0)331 9771696
} 


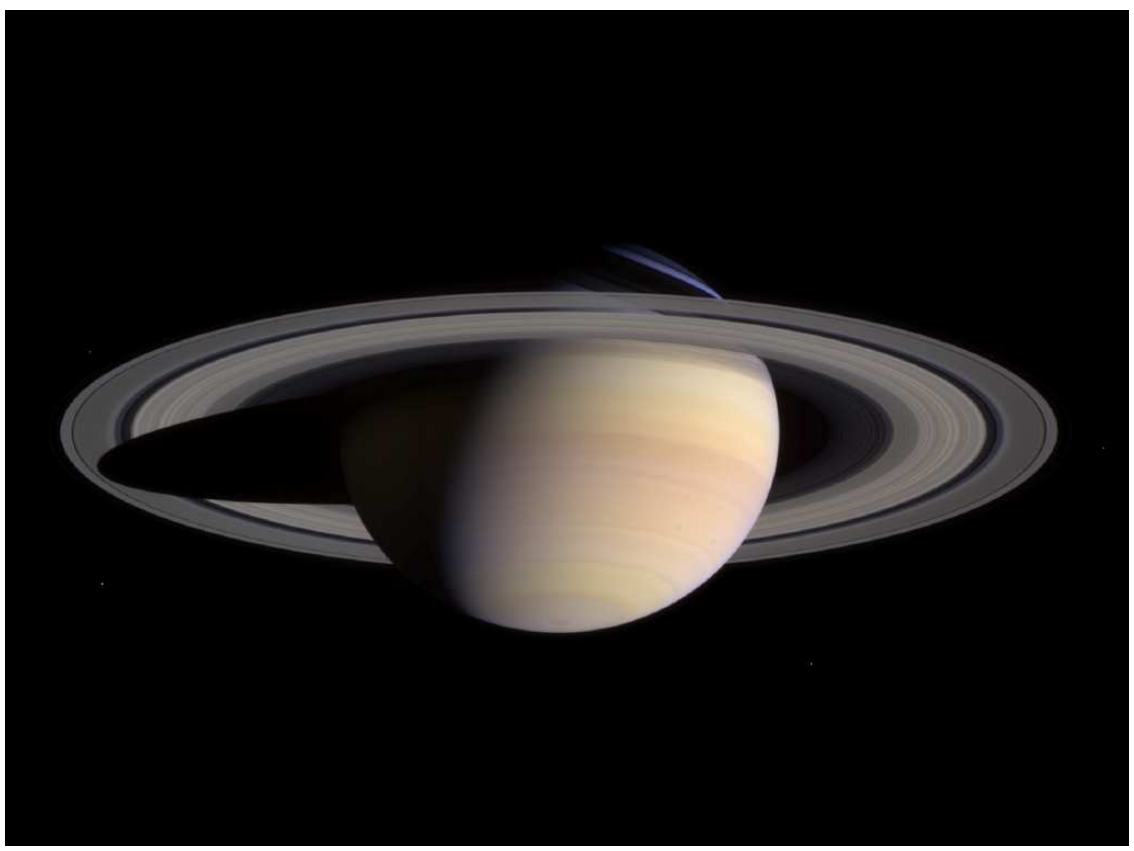

Fig. 1 A ringed world waiting for Cassini: A snapshot of Saturn and its ring system in true color taken by the cameras of the Cassini Spacecraft in May 2004 before the Saturn orbit insertion (SOI: July 1, 2004) from a distance of 28.2 million kilometers. The image shows Saturn and its main rings, A (outermost), B, and C, the Cassini division appearing as the dark band separating the A and B ring.

planets, for instance narrowly confined rings (ringlets), as the F ring, or the ringlets in the Huygens or the Encke gap (see Fig. 2), akin to the rings of Uranus or Neptune. The faint dusty rings of Saturn (E -, G-, and F ring, not visible in the images) bear some resemblance to the rings of Jupiter, or dusty strands in the Neptunian system. Apart from all similarities, Saturn's main rings are unique in their appearance. While the rings of Uranus can be characterized as narrow rings among wide gaps, the rings of Saturn are rather the opposite: narrow voids among extended dense rings.

The large extent of the rings of Saturn, as well as their wealth of structure, is best adumbrated in the Cassini images (Fig.1 and 2). The rings span a distance of about 80,000 kilometers, almost from Saturn's cloud tops at a distance of $60,300 \mathrm{~km}$ from the planetary center, to the outer edge of the A ring at 137,000 kilometers. In contrast to the huge lateral extent the vertical thickness of Saturn's rings is probably on the order of 100 meters only, perhaps even smaller. Thus, planetary rings are about 1000 times thinner than a razor blade, probably the flattest cosmic structures known to date.

The amazing flatness is a result of frequent dissipative collisions among the ring particles. Imagine a particle which is displaced out of the mean ring plane and released into an inclined orbit. Such a grain must cross the ring plane twice, and thus, it will probably suffer collisions with the other ring particles. In this way the vertical component of the relative speed between the ring and the displaced particle is damped out efficiently. The damping settles at a velocity 

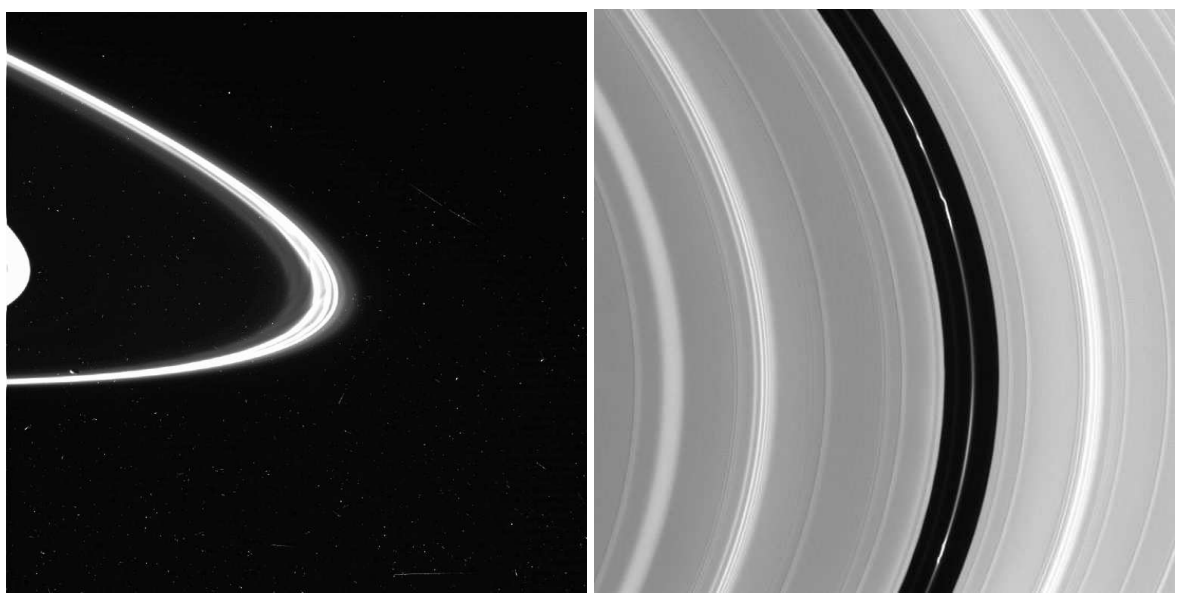

Fig. 2 Narrow rings - ringlets - of Saturn: left: Saturn's F ring shepherded by the satellites Pandora and Prometheus. Right: The A ring with the Encke gap (the black band dividing the figure) which accommodates the small satellite Pan. Around the orbit of the latter a narrow ringlet is observed which shows a similar global morphology as the F ring - bright dots, braids and lumps. Additionally, many wave trains are visible in the A ring. They are identifiable by the brighter stripes in the ring. Images showing waves at a higher resolution are displayed in Fig. 3.

dispersion ("random walk" speed) of the order of $c \approx \Omega D$, where the mean particle diameter is labeled by $D$ and the Kepler frequency $\Omega=\sqrt{G M / a^{3}} \approx 2 \cdot 10^{-4} s^{-1}$. Here, $G$ is the gravitational constant, $M$ the mass of the planet, and $a$ is the semi-major axis of the particle orbit between collisions. With this "random walk" speed the ring particles can only travel a few diameters out of the plane during one orbital period resulting in the amazingly low ring thickness, depending on the mean size of the ring particles. A hydrodynamic justification of this effect is given in Section 2.3.

The Saturnian main rings are composed of macroscopic particles ranging in size from micrometers to house-sized boulders. In the range of a few centimeters to a few meters the sizes of the ring particles satisfy approximately a power law distribution $n \approx\left(D_{0} / D\right)^{3}\left(D_{0}\right.$ - reference size), this size window being responsible for the optical properties of the dense A and B rings of Saturn. First measurements of the size distribution have been performed using the Voyager main gain antenna (RSS-experiment) and have been confirmed by stellar occultation data [15].

Spectral investigations using Voyager-, Hubble-Space Telescope (HST), and recently by the Cassini data (UVIS, VIMS, CIRS) point to an icy composition (water ice contaminated with silicates) of the particles in the main rings. The mechanical properties of such "dirty", frost-covered ice at low temperature have been studied in the laboratory [5, 42]. It was found that small impact speeds, on the order of millimeters to centimeters per second, may lead to a fairly high energy loss in a collision, yielding restitution coefficients $\epsilon$ considerably smaller than one. The coefficient of restitution is defined as the ratio of the normal components of the 

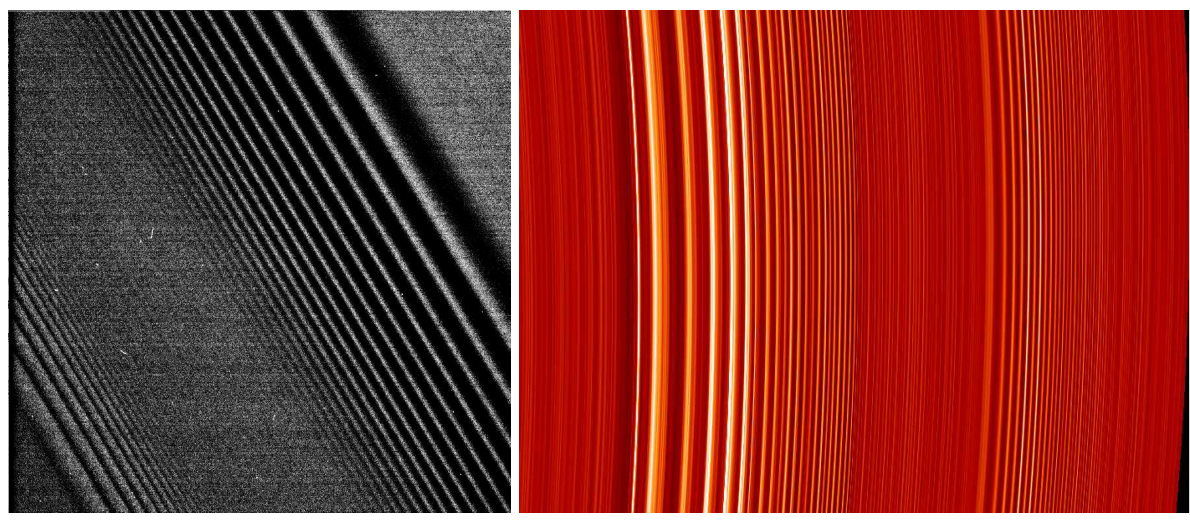

Fig. 3 Rings full of waves: Left: A density wave (lower left corner of the image) excited by the Saturnian moon Prometheus and a bending wave caused by the moon Mimas, in an image taken with the Cassini-cameras. Bending waves are vertical distortions of the mean ring plane generated by an inclined orbit of the satellite. This causes a wave-like warping of the ring visible for the Cassini cameras. Right: Two density wave trains excited by the moon Janus (left and stronger wave) and shepherd Pandora (faint wave in the right part of the image). This image was processed using the UVIS-occultation data (described in the text).

relative velocity of the colliding particles after and before the collision, so that $g_{N}^{\prime}=\epsilon g_{N}$. Visco-elastic particle models $[6,41]$ can be used to fit the function $\epsilon\left(g_{N}\right)$ in the velocity range of $\mathrm{mm} / \mathrm{sec}$ to a few $\mathrm{cm} / \mathrm{sec}$ found in the experiments. The contact dynamics of the particle collision may crucially influence the dynamics, transport processes, and the stability of the rings. In this paper we will use hydrodynamic balance equations to investigate ring instabilities. For more information on the kinetic theory of planetary rings we refer to the original papers [44, 18, 39, 3, 1, 2, 20].

Dissipative collisions together with ring-satellite interactions, both causing radial transport of material and angular momentum, as well as cosmic erosion due to micrometeorites limit the life time of planetary rings to $10^{7} \ldots 10^{8}$ years only. This is considerably smaller than the age of the Solar system, and thus, rings have probably not formed simultaneously with the planets and their satellites. The particulate nature, the composition of the rings, and the rapid cosmic erosion of the ring matter, all point to a relatively "recent" catastrophic disruption of a parent body - for instance a comet [10] or a satellite of the system [8]. Imagine that the comet Shoemaker-Levi 9 would not have hit the planet Jupiter but one of its icy satellites and shattered itself as well as the target satellite. The debris would form a particle cloud of an initially small radial and azimuthal extension. However, the small systematic differences in orbital velocity (Keplerian shear), radially across the particle cloud, would lead to a quick azimuthal spreading of the material. In this scenario a power law size distribution is expected with a few large remainders of the catastrophic disruption, which are almost of the order of the size of the parent bodies. This means, it is very likely that a rare number of moonlets in the size range of tens of meters to kilometers may exist among the majority of centimeter to meter-sized ring particles. The only example known to date is the satellite Pan in the Encke division of Saturn's A ring. 
The armada of outer satellites as well as embedded moonlets modify the particle density of the rings with their gravity. The most impressive examples of disk satellite interactions are density and bending waves, originated by an interplay between the gravitational forces and the Coriolis force - structures which can be understood using hydrodynamic models. Waves are excited at resonances of outer satellites, locations in the ring where the orbital frequencies of the ring particles and the perturbing satellite form an integer ratio. This causes the gravitational perturbation to occur always a the same phase of the particle orbit, pumping up in this way eccentricities or inclinations, which leads finally to the wave excitation.

The left part of Fig. 3 shows an image taken with the Cassini cameras (imaging-sub-system, abbreviated as ISS) of such waves excited at the 12:11 resonance of the satellite Prometheus and the 5:3 vertical resonance of Mimas (a larger icy satellite farther away from the main rings). The vertical excitation of the ring due to Mimas generates a bending wave which is a vertical warping of the whole ring plane. The grazing sun light then casts shadows onto the ring plane which are nicely visible in the left panel (upper right corner) of Fig. 3. Unlike bending waves, density waves are periodical modifications of the ring density. An example is seen in the lower left corner of Fig. 3. The image has a spatial resolution of about $290 \mathrm{~m}$ per pixel. The right part of the figure shows two density wave trains recorded in an occultation experiment of the ultra-violet imaging spectrometer (UVIS) of Cassini. With this technique the light of a star shining through the ring is measured with the UVIS-photometer producing a one dimensional scan with a high resolution of about 10 meters only. The scan has been processed to show a ring-like appearance. These waves are generated by the satellites Janus and Pandora.

Apart from these perturbed regions and the corresponding structures, it is possible that structure may form through instability of the ring flow. Instabilities may bear the chance to explain the tiniest structures observed by Voyager and by Cassini in Saturn's rings. The instabilities are closely related to the transport properties of dense rings. A main topic of this review are the differences of the transport in a planetary ring from force free granular ensembles or granular matter under terrestrial conditions.

In the next section a brief description of the kinetic theory of granular matter and the related hydrodynamic approach is given. The stability of planetary rings is discussed in Section 3. Some consequences are adumbrated in the Conclusions.

\section{Hydrodynamic description of dense rings}

\subsection{Elements of kinetic description}

The analytical approach perhaps best suited for a description of the collisional dynamics of a planetary ring is the kinetic theory for dense systems of hard spheres. It is based on Boltzmann's equation for the evolution of the one particle distribution function $f(\vec{r}, \vec{v}, t)$, extended by Enskog to include effects of the finite particle volume ${ }^{1}$. For a more detailed description the reader is referred to the monographs by Chapman \& Cowling or Résibois \& De Leener $[7,28]$.

The ultimate goal of the kinetic theory is to derive evolution equations for the mean density $n(m, \vec{r}, t)$, momentum $m n(m, \vec{r}, t) \vec{u}(m, \vec{r}, t)$, and energy $T$ of the system ( $T$ - granular

\footnotetext{
1 All variables are listed in table 1
} 
temperature; Boltzmann's constant is normalized to $k_{B}=1$ ). The Boltzmann equation employs aspects as different as the purely mechanical and deterministic two-body interaction of particles and probabilistic assumptions on the distribution function (DF) and the statistical properties of the particle ensemble. Boltzmann defines the one particle DF so that

$$
f(m, \vec{r}, \vec{v}, t) \mathrm{d} m \mathrm{~d}^{3} r \mathrm{~d}^{3} v
$$

is the number of particles of mass $m$ at time $t$ in the volume increment $\mathrm{d}^{3} r$ about $\vec{r}$ and the velocity increment $\mathrm{d}^{3} v$ about $\vec{v}$. The $6 N$-dimensional space spanned by the coordinates $\vec{r}_{i}$ and velocities $\vec{v}_{i}$ of all $N$ particles in the system is called $\Gamma$-space in statistical mechanics. In contrast, the one-particle phase space $(\vec{r}, \vec{v})$ is called $\mu$-space. The increment $\mathrm{d}^{6} \mu=\mathrm{d}^{3} r \mathrm{~d}^{3} v$ is assumed to be small compared to the variations of macroscopic properties of the system but sufficiently large to contain a large number of particles. Additionally, a variable particle mass (adhesion and disruption of particles) can be taken into account. The distribution function can then be regarded as a smoothly varying function of the coordinates $m, \vec{r}$ and $\vec{v}$, and the time evolution of $f$ is governed by the Boltzmann equation

$$
\left(\frac{\partial}{\partial t}+\vec{v} \cdot \frac{\partial}{\partial \vec{r}}+\dot{\vec{v}} \cdot \frac{\partial}{\partial \vec{v}}\right) f(m, \vec{r}, \vec{v}, t)=\left\langle\frac{\partial f(m, \vec{r}, \vec{v}, t)}{\partial t}\right\rangle_{\text {coll }}
$$

where

$$
\dot{\vec{v}}=\frac{\overrightarrow{\mathcal{F}}}{m}
$$

denotes the acceleration due to external forces. One might also expect a term containing the mass variations $\dot{m}$, but such changes only arise in particle collisions (by e.g. fragmentation or adhesion [40]), which enter the collision term. Furthermore, in the following we consider only unbreakable identical hard spheres so that a collision does not change the particle number.

Perturbations arising from the gravitational forces of the ring on the ring particles and those exerted by the numerous satellites orbiting outside or embedded in the rings are important for the structure formation in dense planetary rings. The outer forces can be written as

$$
\overrightarrow{\mathcal{F}}=-\nabla\left(V_{P}+V_{D}+V_{S}\right)+\overrightarrow{\mathcal{F}}_{\text {Dust }}
$$

including the gravity fields of the planet, the rings, and perturbing satellites, $V_{P}, V_{D}$, and $V_{S}$, respectively. The gravitational potential is governed by the Poisson equation

$$
\begin{aligned}
\Delta V & =4 \pi G \rho(\vec{r}, t) \\
\rho(\vec{r}, t) & =\rho_{D}(\vec{r}, t)+\sum_{(S)} M_{S} \delta\left(\vec{r}-\vec{r}_{S}\right)+\rho_{P},
\end{aligned}
$$

with the mass densities $\rho_{D}$ of the ring and $\rho_{P}$ the planet, respectively, and the masses $M_{S}$ of the perturbing satellites.

Dissipative forces $\overrightarrow{\mathcal{F}}_{\text {Dust }}$, relevant for small particles $(O(1 \mu m))$, are the Lorentz force, radiation forces and plasma-dust interactions (drag). Since we focus on the main rings consisting of macroscopic particles larger than a few centimeters in size, these effects are not considered in this review.

In the next section we give a sketch of the construction of the collision integral responsible for the establishment of the equilibrium and the transport processes in planetary rings. 


\subsubsection{The collision integral}

In analogy to the master equation in statistical physics, the collision integral expresses the balance, [7],

$$
\left\langle\frac{\partial f}{\partial t}\right\rangle_{\text {coll }}=G-L
$$

of the number of particles scattered into $(G)$ and those scattered out $(L)$ of the phase space element $\mathrm{d}^{6} \mu$ under consideration. In order to specify these numbers, the following assumptions are beneficial

i) "molecular chaos" apart from collisions;

ii) the ring particles form a dilute gas - i.e. collisions are binary;

iii) the ring particles are inelastic hard spheres.

Assumptions i) and ii) guarantee independent motions of all particles - apart from the collision itself. Thus, the two-particle distribution function can be written as

$$
f^{(2)}\left(\vec{\mu}_{1} ; \vec{\mu}_{2}\right) \approx f\left(\vec{\mu}_{1}\right) f\left(\vec{\mu}_{2}\right)=f_{1} f_{2} .
$$

In other words, higher correlations do not play a role in a dilute gas. The second condition further means that the size of the particles $D$ is negligible compared to the mean free path $\langle l\rangle$, i.e. the location of the collision is well characterized by a point $\vec{r}$.

Assumption $\mathrm{iii}$ ) permits to simply describe the collision dynamics via the cross-section $\sigma_{c}=D^{2} / 4$ and the coefficient of restitution $\epsilon$

$$
\vec{g}^{\prime} \cdot \vec{k}=-\epsilon \vec{g} \cdot \vec{k}
$$

which measures the damping of the normal $(\vec{k})$ component of the relative velocity between the colliding partners $\vec{g}=\vec{v}_{2}-\vec{v}_{1}$. The unit vector $\vec{k}$ labels the direction connecting the centers of either particles and the prime characterizes the post-collisional values.

With these assumptions, the number of collisions between particles per unit time can be written as

$$
\frac{D^{2}}{4} \mathrm{~d}^{3} v_{2} \mathrm{~d}^{2} k(\vec{g} \cdot \vec{k}) f_{1} f_{2}
$$

At this rate particles are leaving the interval $\mathrm{d}^{6} \mu_{1}$ so that the expression for the loss $L$ is found after the five dimensional integration over the velocities $\vec{v}_{2}$ of the collision partners and over the sphere $\mathrm{d}^{2} k$.

The gain term looks similar, with the only difference that one has to find pre-collisional conditions $\left(\vec{g}^{*} ; \vec{k}\right)$ which lead to a scattering into the considered interval $\mathrm{d}^{6} \mu_{1}$. For this purpose, the normal relative impact velocity has to be larger to compensate the damping caused by the dissipation

$$
\left|\vec{g}^{*} \cdot \vec{k}\right|=\frac{1}{\epsilon}|\vec{g} \cdot \vec{k}|
$$

Additionally one has to consider a pre-collisional phase space volume which is larger by the factor $\epsilon^{-1}$ than that for the loss; in this way we account for the dissipation in the system. 


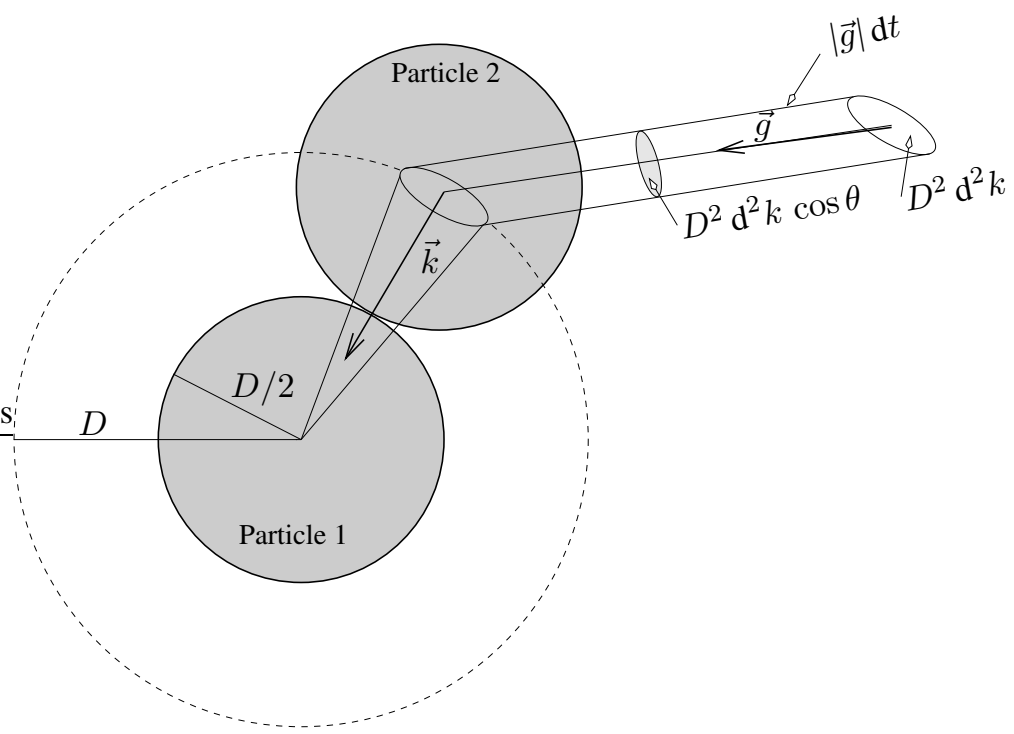

Fig. 4 Collision of two particles of diameter $D$

Having these differences in mind, the gain term looks like relation (10) except that $f_{1} f_{2}$ is replaced by $\left(f_{1}^{*} f_{2}^{*}\right) / \epsilon^{2}$ so that the balance (7) reads now

$$
\begin{aligned}
& \left\langle\frac{\partial f\left(\vec{v}_{1}\right)}{\partial t}\right\rangle_{\text {coll }}= \\
& D^{2} \iint \mathrm{d}^{3} v_{2} \mathrm{~d}^{2} k(\vec{g} \cdot \vec{k}) \Theta(\vec{g} \cdot \vec{k})\left[\frac{f_{1}^{*} f_{2}^{*}}{\epsilon^{2}}-f_{1} f_{2}\right] .
\end{aligned}
$$

The notation $f_{1}^{*}$ is used to denote in short $f\left(\vec{\mu}_{1}^{*}\right)$. The Heaviside function $\Theta(\vec{g} \cdot \vec{k})$ guarantees that only particle pairs are taken into account which are in the process to collide.

The integral (12) does not take into account the finite size of the particles $D$ which become important for Knudsen numbers $k_{n}=D /\langle l\rangle \approx 1$, i.e. if the size $D$ becomes comparable to the mean free path of the ring particles (assumption ii) is lifted). In such a case, the arguments of the function $f$ of the collision partners must be taken at their true centers. So $f_{2}(\vec{r})$ is replaced by $f_{2}(\vec{r}-D \vec{k})$ and correspondingly the gain term $f_{2}^{*}(\vec{r})$ is replaced by $f_{2}^{*}(\vec{r}+D \vec{k})$.

$$
Y_{E}[n(\vec{r} \pm D \vec{k} / 2)]
$$

accounting for an increase of the collision frequency caused by the reduced volume available for the particle motion. With these factors and the modified arguments, the Enskog collision 
integral reads

$$
\begin{aligned}
& \left\langle\frac{\partial f\left(\vec{v}_{1}\right)}{\partial t}\right\rangle_{\text {coll }}=D^{2} \iint \mathrm{d}^{3} v_{2} \mathrm{~d}^{2} k(\vec{g} \cdot \vec{k}) \Theta(\vec{g} \cdot \vec{k}) \times \\
& \times\left\{Y_{E}\left[n\left(\vec{r}_{1}+\vec{k} D / 2\right)\right] \frac{f_{1}^{*}\left(\vec{r}_{1}\right) f_{2}^{*}\left(\vec{r}_{1}+D \vec{k}\right)}{\epsilon^{2}}\right. \\
& \left.-Y_{E}\left[n\left(\vec{r}_{1}-\vec{k} D / 2\right)\right] f_{1}\left(\vec{r}_{1}\right) f_{2}\left(\vec{r}_{1}-D \vec{k}\right)\right\} .
\end{aligned}
$$

In this equation a serious complication appears which we will only reference for completeness. The Enskog factor $Y_{E}$ depends on the particle number density, i.e. on one of the moments of the DF $f$. This means that mathematically we have to deal with a functional in the integrand of an integro-differential equation. Usually an average value $Y_{E}[\langle n\rangle]$ is taken which characterizes the whole system in order to avoid this considerable mathematical difficulty.

\subsection{Hydrodynamic balances}

The next step in the derivation of the macroscopic equations is to take mean values, derived from the one-particle $\operatorname{DF} f(\vec{\mu})$

$$
\langle n \Phi\rangle=\int \mathrm{d}^{3} v \Phi(\vec{r}, \vec{v}) f(\vec{r}, \vec{v}) .
$$

Here $\Phi(\vec{r}, \vec{v})$ stands for certain physical quantities of a particle, e.g. the mass $m$ or momentum $m \vec{v}$. The corresponding collisional changes of the mean $\langle n \Phi\rangle$ are obtained by applying the operator $\int \mathrm{d}^{3} v \Phi(\vec{r}, \vec{v}) \ldots$ to the collision integral (12) to give, [28],

$$
\left\langle\frac{\partial n \Phi}{\partial t}\right\rangle_{\text {coll }}=D^{2} \iint \mathrm{d}^{3} v_{1} \mathrm{~d}^{3} v_{2} \mathrm{~d}^{2} k(\vec{g} \cdot \vec{k}) \Theta(\vec{g} \cdot \vec{k})\left[\Phi_{1}^{*}-\Phi_{1}\right] f_{1} f_{2} .
$$

For the balances of mass and momentum this integral vanishes because in the model of hard, unbreakable spheres the particle number and momenta are not altered by the collision.

With the definitions of mean values and their collisional changes one can formulate a general evolution equation for the mean of $\Phi(\vec{r}, \vec{v})$ based on the kinetic equation (12) or (14)

$$
\left\langle\frac{\partial n \Phi}{\partial t}\right\rangle+\nabla \cdot\langle n \vec{v} \Phi\rangle=n\left\{\langle\vec{v} \nabla \Phi\rangle+\frac{\vec{F}}{m}\left\langle\frac{\partial \Phi}{\partial \vec{v}}\right\rangle\right\}+\left\langle\frac{\partial(n \Phi)}{\partial t}\right\rangle_{\text {coll }} .
$$

Then, for $\Phi=m ; m \vec{v}$ and $\frac{1}{2} m \vec{c} \cdot \vec{c}$ (velocity fluctuation $\vec{c}=\vec{v}-\vec{u}$ ) one is, via multiplication of the Boltzmann equation with $\Phi$ and subsequent integration over the velocity space, led to the balances of mass, momentum and energy. This provides a bridge to phenomenological hydrodynamics

$$
\begin{aligned}
& \frac{\partial \rho}{\partial t}+\nabla \cdot(\vec{u} \rho)=0, \\
& \rho \frac{D \vec{u}}{D t}=\nabla \cdot \mathbf{P}+\vec{f} \\
& \frac{3}{2} \rho \frac{D T}{D t}=-\nabla \cdot \vec{Q}+\mathbf{P}: \varepsilon-\gamma .
\end{aligned}
$$


The substantial derivative is defined by $\frac{D}{D t}:=\partial_{t}+\vec{u} \cdot \nabla$, and the particle number density $n$, the mean velocity $\vec{u}$ and the granular temperature $T$ are given by

$$
n=\int \mathrm{d}^{3} v f(\vec{v}) ; \vec{u}=\frac{1}{n} \int \mathrm{d}^{3} v \vec{v} f(\vec{v}) ; T=\frac{1}{3} \operatorname{Tr} \int \mathrm{d}^{3} v \vec{c} \cdot \vec{c} f(\vec{v}) .
$$

The crucial link is obtained by the constitutive relations - the connection between the momentum and energy fluxes, $\mathbf{P}$ and $\vec{Q}$, and the gradients $\nabla \rho, \nabla T$, and

$$
\varepsilon=\frac{1}{2}[\nabla \vec{u}+\vec{u} \nabla]
$$

where the mass density is $\rho=m n$. These relations are necessary in order to properly close the system (17)-(19). A term appearing in granular matter is the dissipation rate due to the inelasticity of the collisions

$$
\gamma=\langle\dot{T}\rangle_{\text {coll }} \propto\left(1-\epsilon^{2}\right)
$$

The constitutive relations are derived, for instance, by expansions of $f$ about the quasiequilibrium ( $f_{0}-$ Maxwellian). One method is found by the Chapman-Enskog expansion, carried out with respect to the thermodynamic "forces" - the gradients of the macroscopic state-variables: $\nabla n ; \nabla \vec{u}$ and $\nabla T$. In this context, the zeroth and the first order approximations are named after Euler and Navier-Stokes, respectively. From the latter, e.g. the shear viscosity $\eta$ can be derived.

In the next subsection, we will employ an empirical method $[19,21]$ in order to motivate a dependence of the shear viscosity $\eta=m n \nu$ on the above state variables, rather than retracing very technical expansions of $f$ or alternative approaches of comparable complexity.

\subsection{Constitutive relations}

The link between kinetics and hydrodynamics is given by the closure of the system of equations: i.e. the connection between the gradients $\nabla \vec{u}$ and $\nabla T$ and the related fluxes of momentum $\mathbf{P}$ (stress tensor) and energy $\vec{Q}$. Linear relations - the Fourier and the Newton law $-$

$$
\begin{aligned}
& \vec{Q}=-\kappa \nabla T \\
& \mathbf{P}=-p \mathbf{I}+2 \eta \varepsilon-\left(\frac{2}{3} \eta-\zeta\right) \nabla \cdot \vec{u}
\end{aligned}
$$

correspond to the Navier-Stokes order of the Chapman-Enskog expansion [28]. As mentioned above, the linear coefficients, heat conductivity $\kappa$ and the shear viscosity, $\eta$, can be deduced from kinetics by performing the Chapman-Enskog expansion up to the Navier-Stokes order (linear in $\nabla T$ and $\nabla \vec{u}$ ) yielding Eqs. (23) and (24). The bulk viscosity arises in a dilute system if rotational or vibrational degrees of freedom are taken into account in the $\operatorname{DF} f(\vec{r}, \vec{v}, \vec{\omega})$, or, in a dense system as a consequence of the finite particle volume [7].

In general, in a granular gas expressions are obtained for the viscosity $\eta$ and the heatconductivity $\kappa$ that differ considerably from those that are appropriate for a planetary ring. This means, that the form of the transport coefficients depends on the physical conditions of the system under consideration. This is different from fluid dynamics, where the transport coefficients are material properties. The major reason of the complexity in the case of granular matter is the lack of a clear separation between the interparticle scale $D$, the mean free path $\langle l\rangle$, 
and the spatial dimension on which the state variables experience a considerable change. In the case of ordinary gases, or fluids, these length scales are separated by orders of magnitude. In granular gases and in dense planetary rings they may be all of the same order of magnitude.

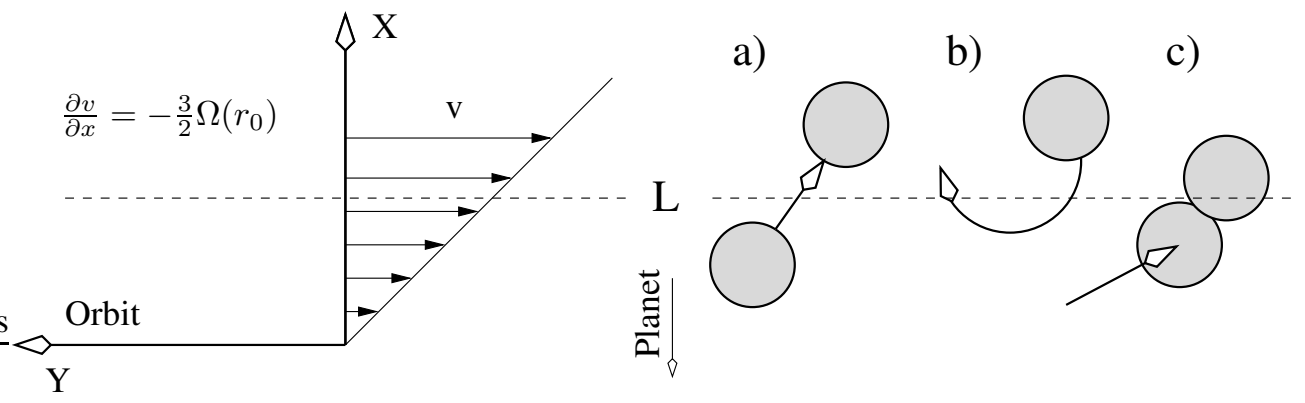

Fig. 5 Left panel: Stream velocity of the ring in a local frame co-rotating at a given distance from the planet with angular velocity $\Omega\left(r_{0}\right)$. The $\mathrm{X}$-axis points radially outward, the Y-axis in the direction of the orbit. Right panel: Typical particle paths and collisions. a) Momentum transport across the imagined line $L$ by a crossing particle (local transport); b) particle moving on an epicycle (no transport); c) momentum transport across $L$ in a collision (non-local transport).

In view of the crucial differences between granular and usual molecular gases, we follow here the strategy by Hwang \& Hutter [21], who deduced relations for the transports using dimensional arguments for dense granular matter. We give an expression for the shear viscosity $\eta$ and analogous relations for the bulk viscosity $\zeta$ and the heat conductivity $\kappa$.

Before presenting the procedure, we discuss the different contributions to the transports illustrated in Fig. 5. There are basically two processes transporting energy and momentum over a characteristic radial distance $l_{c}$. One is given by the mere "free" motion of a particle crossing an arbitrary radial location labeled here by the dashed line $L$. The particle carries momentum and energy over the characteristic radial distance, which is the mean free path in this case $l_{c}=\langle l\rangle$. This transport is simultaneously related to a transfer of matter crossing the line $L$. In the dynamics of planetary rings it is called the local contribution because the small distance of particle centers at the moment of the collision is not taken into account. In the literature on the kinetics of dense hard spheres the related transport is called molecular. The second process is transport of momentum and energy from particle center to particle center in a collision, over one particle diameter $l_{c}=D$. In this case the transports are not related to mass transfer or particle motions, rather to internal stresses of the particle material. In the planetary community this transport contribution has been named non-local, in the literature on hard sphere kinetics it is labeled collisional transfer.

We introduce a rectangular coordinate system $\vec{r}=x \vec{e}_{x}+y \vec{e}_{y}+z \vec{e}_{z}$, co-rotating with the ring material at a given radial distance $r_{0}$ from the planet. The radial coordinate $x=r-r_{0}$ is measured from $r_{0}$ radially outward and the $y$-axis points in the direction of a circular orbital motion $\left(\vec{e}_{y}=\vec{e}_{\phi}\right.$, see Fig.5). This coordinate system is advantageous if small spatial distances are considered, i.e. $|x| \ll r$. Using the Keplerian frequency $\Omega(r)=\sqrt{G M / r^{3}}$ we can then linearize the Keplerian velocity field $\vec{u}(r)=\Omega(r) r \vec{e}_{y}$ in powers of $x$, so that

$$
\vec{u}=-\frac{3}{2} x \Omega_{0} \vec{e}_{y}
$$


where $\Omega_{0}=\Omega\left(r_{0}\right)$. In what follows we restrict attention on axisymmetric ring configurations, so that all variables only depend on $x$.

The local transport is characterized by the amount of (angular) momentum transported radially outward by particles. Due to the systematic shear each particle coming from smaller distances to Saturn carries a bit excess momentum compared to those revolving further away from the planet. The coefficient of viscosity is defined as the factor of proportionality between the stress $P_{x y}$ and the shear rate $-(3 / 2) \Omega_{0}$. In order to estimate the number of particles crossing the line $L$ per time unit we assume a given mean free path $\langle l\rangle$ and a dispersion velocity $c=\sqrt{T}$, which will be specified later. The momentum density is given by $\overrightarrow{\mathbf{p}}=$ $m n(x) v(x) \vec{e}_{y}$, so that the flux of momentum in the $x-y$ component reads $P_{x y} \approx c \Delta \mathbf{p}$, where the excess of momentum $\Delta \mathbf{p}$ has to be calculated for the characteristic transport length $l_{c}=$ $\langle l\rangle$. This momentum excess is obtained by expanding the momentum density and recalling that $\partial_{x} v(x)=-(3 / 2) \Omega_{0}$

$$
\Delta \mathbf{p}=m\left[v(x) \partial_{x} n(x)+n(x) \partial_{x} v(x)\right]_{x=0}\langle l\rangle,
$$

implying for an unperturbed disk with $\partial_{x} n(x)=0$

$$
P_{x y}=-\frac{3}{2} n(x) m c\langle l\rangle \Omega_{0}=-\frac{3}{2} \rho c\langle l\rangle \Omega_{0} .
$$

Recalling the dynamic viscosity $\eta$ to be the factor of proportionality between momentum flux $P_{x y}$ and shear rate $-(3 / 2) \Omega_{0}$ we are left with the classical expression

$$
\begin{aligned}
P_{x y} & =\rho c\langle l\rangle \frac{\partial v(x)}{\partial x}, \\
\eta & =\rho c\langle l\rangle \\
& =\rho \frac{c^{2}}{\omega_{c}}
\end{aligned}
$$

where $\omega_{c}=c /\langle l\rangle$ is the collision frequency. For the local kinematic viscosity $\nu=\eta / \rho$ we recover the expression

$$
\nu_{l}=\langle l\rangle^{2} \omega_{c} .
$$

However, this expression for the local viscosity is valid only for dense systems. In dilute systems the curved motion of particles between collisions has to be taken into account. In addition, the adjustment of the scale hight of a planetary ring with the velocity dispersion, or equivalently, the dependence of the collision frequency $\omega_{c}$ on the density, or on the optical depth of the ring, has to be considered. The optical depth is defined as

$$
\tau(x)=\pi \frac{D^{2}}{4} \int_{-\infty}^{\infty} \mathrm{d} z n(x, z) .
$$

To fix ideas we employ a Gaussian vertical profile. It can be derived from the stationary isothermal vertical $(z)$ momentum balance

$$
\frac{1}{\rho} \frac{\partial p}{\partial z}=-\frac{\partial V}{\partial z} \approx-\Omega^{2} z
$$


and the equation of state

$$
p \approx \rho c^{2}
$$

to give finally

$$
n(z)=n(0) \exp \left(-\frac{\Omega^{2} z^{2}}{2 T}\right)
$$

if the velocity dispersion $c$ is assumed to be constant. With this the optical depth can be written as

$$
\tau=\left(\frac{\pi}{2}\right)^{3 / 2} D^{2}\langle H\rangle n(0) \approx D^{2} n_{s},
$$

in which the half-thickness of the ring is defined by

$$
\langle H\rangle=\sqrt{T} / \Omega \propto c / \Omega
$$

and the surface number density is given by $n_{s}=2 n(0)\langle H\rangle$. With the latter the surface mass density is defined by $\sigma=m n_{s}$.

It is worth mentioning that the half-thickness of a planetary ring $\langle H\rangle$ is only about a few typical particle diameters, i.e. only a few meters compared to hundred-thousands of kilometers in lateral direction. Thus, in the following vertically integrated quantities will be considered ${ }^{2}$ : mass density $\sigma$ (optical depth $\tau$ ), pressure $p$, pressure tensor $\mathbf{P}$, and transport coefficients $\eta, \zeta$, and $\kappa$. As a consequence of this averaging the pressure $p$ (dynamic viscosity $\eta$ ) measures a force (momentum) per unit length instead per unit area. Furthermore, only dimensional analyses are performed which will reproduce the results of very technical expansions of the kinetic equations $[18,3]$ with a rather simple treatment.

With the values (32)-(36) let us reconsider the expression for the kinematic shear viscosity from Eq. (31) $\nu \approx \omega_{c}\langle l\rangle^{2}$. First we estimate the collision frequency $\omega_{c}$, which can be approximated as the number of collisions per unit time

$$
\omega_{c} \approx \pi c D^{2} n(x, z) \approx \pi D^{2} c \frac{n_{s}}{\langle H\rangle} \sim \pi D^{2} \Omega n_{s} \sim \Omega \tau .
$$

This is a very important result because the collision frequency $\omega_{c}$ does not depend on the granular temperature $c \propto \sqrt{T}$ due to a vertically balancing disk with $\langle H\rangle \propto c / \Omega$. This is an important difference between usual gases/granular gases in a force-free environment and those composing a Keplerian disk around a central body. The second difference comes from the expression for the mean free path $\langle l\rangle$ which, under force-free conditions, diverges for $n \rightarrow 0$. This is not the case for particles orbiting a central body. Since particles move on Keplerian ellipses their radial motion is principally restricted. Thus, the maximal radial excursion which a particle can make in the co-moving coordinate system is the epicyclic length $\langle l\rangle_{\max } \approx c / \Omega$. Thus, in the dilute limit we obtain for the kinematic local viscosity

$$
\nu_{l}(n \rightarrow 0) \stackrel{(31)}{=} \omega_{c} \frac{c^{2}}{\Omega^{2}} \text { for } \Omega \gg \omega_{c}
$$

\footnotetext{
${ }^{2}$ No new symbols will be introduced in order to restrict the number of symbols.
} 
This expression can be combined with equation (31) to yield, [18],

$$
\nu_{l}=c^{2} \frac{\omega_{c}}{\omega_{c}^{2}+\Omega^{2}}=\frac{c^{2}}{\Omega} \frac{\tau}{1+\tau^{2}} .
$$

The nonlocal contribution to the kinematic viscosity is somewhat simpler to describe (see Fig. $(5 \mathrm{c}))$. Instead of the mean-free path $\langle l\rangle$, the transports have to bridge only the particle diameter $D$. Thus, instead of $c\langle l\rangle$ one may write $\omega_{c} D^{2}$ in Eq. (29), and then obtains with (38)

$$
\nu_{n l}=D^{2} \omega_{c} \propto D^{2} \Omega \tau \text {. }
$$

This expression can easily be understood: the number of particles per unit area and time crossing $L$ is $n c$, that fraction laying directly on this section is obtained by multiplying with a factor $D /\langle l\rangle$ so that we have $n c D /\langle l\rangle=n \omega_{c} D$. The second factor $D$ comes from the expansion to obtain $\Delta$ p yielding finally Eq. (41) for the nonlocal kinematic viscosity.

Equations (40) and (41) can be combined into one equation for the total kinematic shear viscosity

$$
\nu=\nu_{l}+\nu_{n l}=A \frac{c^{2}}{\Omega} \frac{\tau}{1+\tau^{2}}+B D^{2} \Omega \tau,
$$

with $A$ and $B$ as weighting factors.

For the purposes of a stability analysis (presented in Section 3) we will use a power law

$$
\eta=\tau \nu \approx \alpha \frac{c^{2}}{\Omega} \tau^{1+\beta}
$$

for the vertically integrated dynamic viscosity. Expression (43) is then assumed to be valid locally in the vicinity of a given optical depth $\tau_{0}$, where the slope $\beta$ can be regarded as constant. Consequently, only small or moderate deviations of the optical depth $\tau^{\prime}=\tau-\tau_{0}$ can be treated ( $\tau_{0}-$ optical depth of the steady state).

In a similar way, local and nonlocal contributions can be derived for the heat conductivity and the pressure, [7], to give

$$
\kappa=C_{\kappa} \eta
$$

with a constant $C_{\kappa}$ and

$$
p=\sigma c^{2}\left[1+\frac{\Omega D \tau}{c}\right]
$$

For the bulk viscosity $\zeta$ we simply assume the same functional dependence as for the shear viscosity but weighted with a factor $C_{\zeta}$

$$
\zeta=C_{\zeta} \eta
$$

One term is left to be specified in the hydrodynamic equations - the granular cooling or dissipation rate. For mere translational motions this cooling is given by

$$
\begin{aligned}
\gamma & =\frac{D^{2}}{4} \int \mathrm{d}^{3} \vec{g} \mathrm{~d}^{2} \vec{k}\left\{\frac{m}{2}\left(\vec{g}^{\prime}\right)^{2}-\frac{m}{2}(\vec{g})^{2}\right\} f(\vec{g}) \\
& \approx C_{c} \omega_{c}\left(1-\epsilon^{2}\right) c^{2},
\end{aligned}
$$


where in force-free cases one obtains $\gamma \propto\left(1-\epsilon^{2}\right) c^{3}$ because $\omega_{c} \propto c$. The latter dependence is different in cosmic disks due to the height adjustment explained above so that for planetary rings it follows

$$
\gamma \propto \Omega \tau\left(1-\epsilon^{2}\right) c^{2} .
$$

Finally, we include effects of self-gravity of the ring material using the solution of the Poisson equation for a thin disk. Assuming axisymmetry and $\rho(x, z)=\sigma(x) \delta(z)$ the Poisson equation (5) reduces to

$$
\frac{\partial^{2} V_{D}}{\partial x^{2}}+\frac{\partial^{2} V_{D}}{\partial z^{2}}=4 \pi G \sigma \delta(z) .
$$

For a surface mass density $\sigma(x) \propto \exp [\imath k x]$ the solution is straightforward, [4],

$$
V(x, z)=-\frac{2 \pi G}{|k|} \sigma(x) \exp (-k|z|) .
$$

\section{Stability of dense rings}

A classical problem of ring dynamics is the stability of the ring flow. It addresses dynamical processes on time-scales that are small compared to the secular viscous evolution of the disc. Naturally, small perturbations of the stationary state are unavoidable (for instance through the gravitational force of the satellites of the planet). The question of stability theory is whether the perturbed ring will return to the stationary state, i.e. whether the ring is stable with respect to the perturbation, or, if the perturbation will grow. In the latter case the stationary state is called unstable. If the flow is unstable, the stationary state is destroyed by perturbations of arbitrary smallness. It will eventually be replaced by another configuration which is stable, and, in general more complex and structured.

A strong motivation for the stability analysis of planetary rings was certainly the wealth of radial structures of Saturn's rings revealed by the Voyager observations and topped by the amazing Cassini images. However, many of these features are not correlated to external perturbations (i.e. satellites). This lack of an explanation in terms of external perturbations ${ }^{3}$ stimulated the search for mechanisms of intrinsic instability, that could produce at least part of the observed structure. Prominent models proposed in the literature are the diffusion instability [25, 24, 45], ballistic transport models $[11,13,9,12]$, transport related to electromagnetic effects on small grains [17, 16, 38], viscous overstability [36, 37, 33, 35], or instability of the radial shear profile due to a certain dependence of shear stress on the shear rate of the flow [43].

In the following section we give a brief account of the stability analyses of a thin granular disk in terms of the diffusion instability, also called viscous instability in the literature ([25, $24,45])$, and viscous overstability ([36, 37, 33, 35, 34]).

\subsection{Linear Instability Analysis}

Mathematically, unperturbed planetary rings can be modeled by the system of coupled, nonlinear, partial differential equations (16)-(19), and the closures (23)-(24), (44)-(45), that describe

\footnotetext{
3 Orbital resonances with outer satellites explain many isolated features in Saturn's rings
} 
the evolution of average properties of the flow, such as mass density, radial and tangential momentum, and the energy of the particles' random motion.

Usually the approximation of a thin disk is applied, i.e. a vertical integration is applied and the balance equations (16)-(19) are written for the surface mass density $\sigma$. The pressure and the transport coefficients are then vertically integrated quantities. We write the state variables as one vector, reading in our case

$$
\vec{\Psi}(\vec{r}, t)=\left(\begin{array}{c}
\sigma(\vec{r}, t) \\
\vec{u}(\vec{r}, t) \\
T(\vec{r}, t)
\end{array}\right)
$$

so that the set of model equations can be written in a compact form as

$$
\frac{\partial \vec{\Psi}}{\partial t}=\vec{F}\left(\vec{\Psi}, \vec{\nabla} \vec{\Psi}, \vec{\nabla}^{2} \vec{\Psi}\right) .
$$

We assume the ring in a stationary state that is perturbed. The stationary state $\vec{\Psi}_{0}$ is characterized by zero time-derivatives, so that

$$
\vec{F}\left(\vec{\Psi}_{0}\right) \equiv 0 .
$$

We are interested in the time evolution of a tiny deviation from the stationary solution

$$
\vec{\Psi}(\vec{r}, t)=\vec{\Psi}_{0}(\vec{r}, t)+\delta \vec{\Psi}_{1}(\vec{r}, t) .
$$

The dimensionless parameter $\delta$ is introduced to keep track of different orders of $\vec{\Psi}_{1}$ when equation (54) is inserted in (52). At the level of linear stability it is not necessary to fix $\delta$ except that it is chosen sufficiently small so that orders $\delta^{2}$ can be neglected. Using relation (53) we obtain for the first order in $\delta$

$$
\left(\frac{\partial}{\partial t}-\partial \tilde{\mathbf{F}}\right) \vec{\Psi}_{1}=0
$$

where $\partial \tilde{\mathbf{F}}$ is the Jacobian matrix of the right-hand side $\vec{F}$ of equation (52)

$$
\left.\partial \tilde{\mathbf{F}} \equiv \frac{\partial \vec{F}\left(\vec{\Psi}, \vec{\nabla} \vec{\Psi}, \vec{\nabla}^{2} \vec{\Psi}\right)}{\partial \vec{\Psi}}\right|_{\vec{\Psi}=\vec{\Psi}_{0}}=\left(\begin{array}{ccc}
\partial_{\sigma} F_{1} & \partial_{\vec{u}} F_{1} & \partial_{T} F_{1} \\
\partial_{\sigma} F_{2} & \partial_{\vec{u}} F_{2} & \partial_{T} F_{2} \\
\partial_{\sigma} F_{3} & \partial_{\vec{u}} F_{3} & \partial_{T} F_{3}
\end{array}\right) .
$$

For simplicity we restrict ourselves to axially symmetric perturbations and use the reduced radial coordinate $x=r-r_{0}$. The value $r_{0}$ may be chosen to correspond to a given region in Saturn's rings, in which we are particularly interested.

If the Jacobian does not depend on $t$ and $x$ then equation (55) has solutions of the form

$$
\vec{\Psi}_{1}=\int_{-\infty}^{\infty} \mathrm{d} k \vec{\psi}^{(k)} \exp \{s t+\imath k x\}
$$


with the amplitude vector $\vec{\psi}^{(k)}$, the real wavenumber $k$, the complex growth rate $s$ (the symbol $\imath$ denotes the imaginary unit). Equation (55) then reduces to the eigenvalue problem

$$
s(k) \vec{\psi}^{(k)}=\hat{M}(k) \cdot \vec{\psi}^{(k)}
$$

where $\hat{M}(k)$ is the Jacobian matrix $\partial \tilde{\mathbf{F}}$ in Fourier space. The eigenvalue problem has nontrivial solutions $s$ satisfying the 4 -th order polynomial dispersion relation

$$
\operatorname{Det}[\hat{M}(k)-\widehat{\mathbf{I}} s]=0 \text {. }
$$

The linear instability of the stationary solution $\vec{\Psi}_{0}$ is determined completely by the eigenvalues $s_{j}$. Since equations (52) are real, the coefficients of the dispersion relation (59) are also real. Consequently the eigenvalues $s_{j}$ are either real or they come as complex conjugate pairs. If the real parts of all eigenvalues are negative for all values of the wavenumber $k$, then the stationary state $\vec{\Psi}_{0}$ is stable. If at least one eigenvalue has a positive real part for a range of wavenumbers $k$, then the stationary state is unstable.

The analysis of the eigenvalues of the 4-th order dispersion relation (59) is given in [35] and results are compared with direct particle simulations in [33]. The thermal excitations of the system are found to introduce no qualitatively new effects. They merely shift stability boundaries and modify growth-rates accordingly. To display the principles it suffices to consider an isothermal ring which leads to a considerable simplification of the analysis.

\subsection{Isothermal Systems}

For constant granular temperature the phase space is given by the surface mass density $\sigma(x)$ and the velocities $u(x), v(x)$. In this case the matrix of the eigenvalue problem reads

$$
\hat{M}(k)=\left(\begin{array}{ccc}
0 & -\imath k \sigma_{0} & 0 \\
\imath\left(g-\frac{p_{\sigma} k}{\sigma_{0}}\right) & -k^{2} \nu\left(\frac{4}{3}+\frac{\zeta}{\nu}\right) & 2 \Omega_{0} \\
-\imath \frac{3 \Omega_{0}}{2 \sigma_{0}} \nu(1+\beta) k & -\frac{1}{2} \Omega_{0} & -k^{2} \nu,
\end{array}\right),
$$

with the derivative $p_{\sigma}=\partial p / \partial \sigma$ and $g=2 \pi G$. Here all quantities $\left(\nu, \zeta, c_{0}, \Omega_{0}, p_{\sigma}\right)$ are constants, characterizing the stationary state. For the dependences of shear- and bulk-viscosities on the surface density a parameterization $\propto \tau^{\beta}$ is assumed. We obtain the dispersion relation, first given in [36],

$$
\begin{aligned}
& s^{3}+s^{2} k^{2}\left[\frac{7}{3} \nu+\zeta\right]+s\left[\Omega_{0}^{2}-k g \sigma_{0}+p_{\sigma} k^{2}+k^{4} \nu\left(\frac{4}{3} \nu+\zeta\right)\right] \\
& +\nu k^{2}\left[3 \Omega_{0}^{2}(1+\beta)-k g \sigma_{0}+k^{2} p_{\sigma}\right]=0
\end{aligned}
$$

of third order in the complex growth-rates $s$. We discuss the solutions in terms of the parameter

$$
\beta=\frac{\partial \ln (\nu)}{\partial \ln \sigma}
$$

labeling the density dependence of the kinematic viscosity. The value of $\beta$ is fixed for a given system but it would vary for different ring regions depending on the background surface mass density. The dispersion relation has one real solution and a pair of complex conjugate roots. 


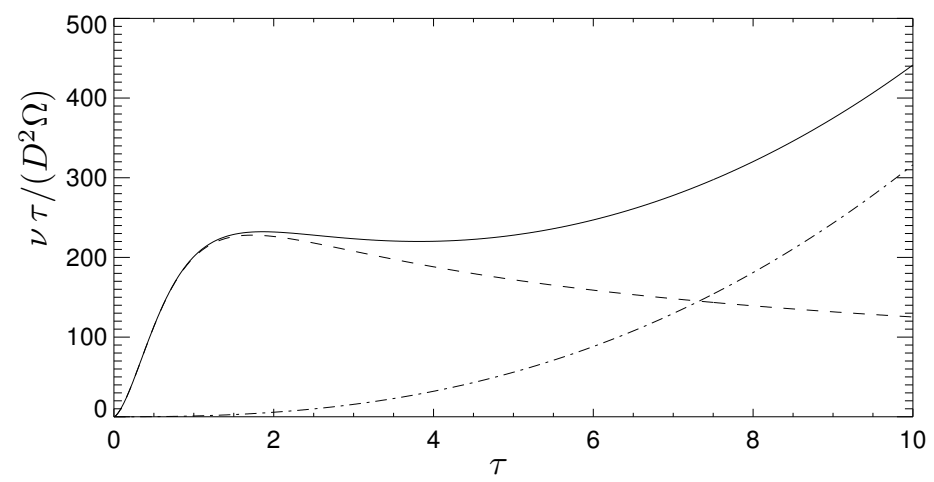

Fig. 6 Qualitative dependence of the dynamic viscosity $\nu \tau$ on the optical depth $\tau$, that could be realized in a disk of fairly elastic particles. The local and nonlocal contributions are shown as dashed and dashdotted lines, respectively.

\subsection{Viscous instability}

The real solution of the dispersion relation (61) reads

$$
s_{1}=-3 \nu(1+\beta) k^{2}+O\left(k^{3}\right)
$$

corresponding to the so-called viscous instability. These modes become unstable for

$$
\beta<-1 \text {. }
$$

The physical picture is that radial diffusion of material is directed towards enhancements of density, so that any density fluctuation is amplified. Equation (42) does not allow for $\beta<-1$ for a constant dispersion velocity $c$. However, if the equilibrium velocity dispersion is a decreasing function of optical depth $\tau$, then the condition for viscous instability can in principle be fulfilled for an intermediate range of values of the optical depth. The dependence of the total shear viscosity on $\tau$ could qualitatively look like in Fig. (6). In this case, if the particles are sufficiently elastic to maintain a fairly high dispersion velocity, for small optical depth the local component of the viscosity is dominant, but will decrease rapidly with increasing $\tau$. The nonlocal component of the viscosity becomes dominant for large optical depth. For a $\nu \tau$ vs $\tau$ relation as shown in Fig. (6) all ring regions with optical depths between roughly 2 and 4 would be viscously unstable. The ring would evolve into a number of radial bands with a bimodal distribution of optical depth.

It must be noted, that the scenario of viscous instability requires rather drastic assumptions on the elastic properties of the particles [25], which are probably not fulfilled in Saturn's rings.

\subsection{Viscous overstability}

Besides a real solution the dispersion relation (61) has a pair of complex conjugate solutions

$$
s_{2,3}= \pm \imath \Omega\left(1-\frac{g \sigma_{0}}{2 \Omega^{2}}|k|+\frac{k^{2}}{2 \Omega^{2}} \frac{\partial p}{\partial \sigma}\right)+\frac{3}{2} \nu k^{2}\left(\beta-\frac{1}{3}\left[\frac{\zeta}{\nu}-\frac{2}{3}\right]\right)+O\left(k^{3}\right)
$$


characterizing viscous overstability. The criterion for instability is in this case

$$
\beta>\frac{1}{3}\left[\frac{\zeta}{\nu}-\frac{2}{3}\right]
$$

with the kinematic shear viscosity $\nu$ and the kinematic bulk viscosity $\zeta$.

In contrast to the viscous instability, viscous overstability is indeed observed in direct particle simulations for parameters that are realistic for the dense parts of the Saturnian ring system. Axisymmetric oscillating structures form spontaneously and start to grow exponentially. Eventually the growth of the amplitudes slows down and the amplitudes saturate. The wavelengths of the modes are typically on the order of 100 particle radii and the oscillation frequency is close to the Keplerian frequency (see imaginary part of equation (64)). The oscillations soon transform into traveling waves.

To investigate the stability properties theoretically we need to fix values for $\nu$ and $\zeta$. To this end values for the unperturbed transport coefficients have been determined in direct particle simulations of a planetary ring [33]. For a description of the simulation method see [46, 29, $30,31,32]$. In these simulations the fluxes of momentum and energy were tabulated for a system of identical meter-sized particles with a velocity dependent coefficient of restitution given by the relation

$$
\epsilon\left(g_{N}\right)=\left(\frac{g_{N}}{g_{c}}\right)^{-0.234},
$$

where $g_{N}$ is the normal component of the impact speed $\vec{g}$. This relation was found in laboratory measurements with water ice by Bridges et al. [5], giving a value of $g_{c}=0.077 \mathrm{~mm} \mathrm{~s}^{-1}$. These simulations were carried out without direct particle self-gravity to suppress the simultaneous appearance of the so called gravitational wakes to study overstability separately. Gravitational wakes are non-axisymmetric structures that form by the tidal disruption of temporarily gravitationally bound clusters of particles (see Fig. (9) for simulational examples). One main effect of self-gravity would be an increase of the collision frequency of the particles. This effect has been taken into account by using in the particles' equations of motion an increased frequency of vertical oscillations $\Omega_{z} / \Omega>1$ compared to the Keplerian frequency. This amounts to an effective inclusion of the disk's mean self-gravity into the vertical component of the planet's gravity. The result is a flattening of the ring and an increase of the collision frequency similar to the one of direct self gravity. Results for the transport coefficients determined from the simulations are shown in Fig. (7). For details see [33].

Using values from Fig.(7) [33], theoretical growth-times of viscous overstability from the dispersion relation (61) and from a 4th-order dispersion relation of a non-isothermal hydrodynamic model have been compared to the growth-times of viscously overstable modes determined in direct simulations [35].

The comparison is shown in Fig. (8). The simulations were carried out for an enhanced vertical frequency $\Omega_{z}=3.6 \Omega$. The plots on the left are for a system with $\tau=1$ those on the right for $\tau=2$. The upper panels show the areas of overstability in the plane spanned by the wavelength and the parameter $\beta$. The dashed horizontal line marks the value of $\beta$ that was actually realized in the simulations, determined from the $\tau$ dependence of the viscosity of the unperturbed system (Fig. (7). The 10-folding times for the amplitudes of oscillations are plotted in the lower panels as functions of the wavelength. Two cases are considered: 


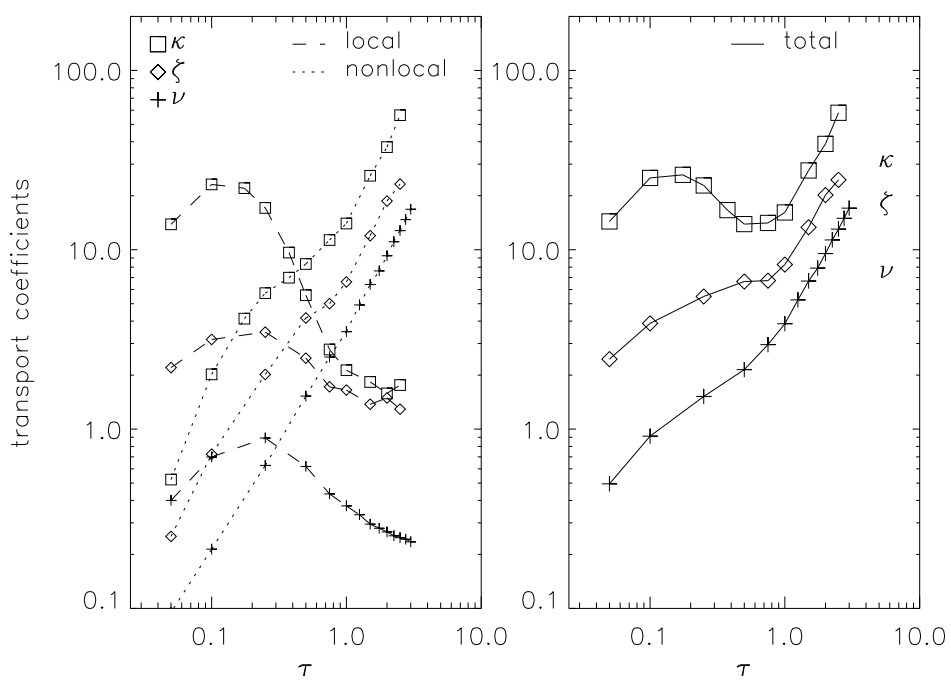

Fig. 7 Kinematic transport coefficients as functions of the optical depth $\tau \propto \sigma$. The unit on the vertical axis is $\Omega D^{2}$. In the left part local (long dashes) and nonlocal (short dashes) contributions are shown separately, whereas in the right panel the total transports are plotted. Besides the shear viscosity $\nu$ the heat conductivity $\kappa$ and the viscosity $\zeta$ are shown, (figure taken from [33]).

the isothermal (dashed lines) and the non-isothermal (solid lines) model. 10-folding times of overstable modes observed in simulations are plotted as diamonds. Crosses correspond to the decay-times of stable modes that were imposed to the system with the initial values. In general a good agreement is found between the non-isothermal model and the simulations. In addition to the growth-times the correct stability boundary, on the order of $100 \mathrm{~m}$, is predicted by the theory. The properties of the eigenfunctions for the perturbed values $(\sigma, u, v)$ have also been confirmed by numerical experiments [35], and a weakly nonlinear analysis [34] has demonstrated the amplitude saturation and preference of traveling waves in the nonlinear state.

For the case of direct particle-particle gravity overstability generally coexists with the nonaxisymmetric so-called gravitational wakes [30, 32]. Gravitational wakes form spontaneously due to gravitational instability as a result of the competing effects of the clustering of ring particles due to self-gravity and disruption by shear. They are the analogues to spiral arms of a galactic disk [23]. The plot shows a series of simulations, each after the evolution of 50 orbital periods. The simulation parameters are $\tau=1.4 ; \sigma=840 \mathrm{~kg} \mathrm{~m}^{-2}$ and $\Omega=2 \cdot 10^{-4} \mathrm{~s}^{-1}$. The four rows correspond to different internal particle densities and particle radii. The values are given in the plot titles in SI - units. In order to keep $\tau$ and $\sigma$ constant, particle numbers in the range $N=15,000 \ldots 60,000$ had to be simulated. Left column: Ring plane seen from above. The length in $x$-direction is about $580 \mathrm{~m}$ and in $y$-direction $230 \mathrm{~m}$. The planet is in $-x$ 

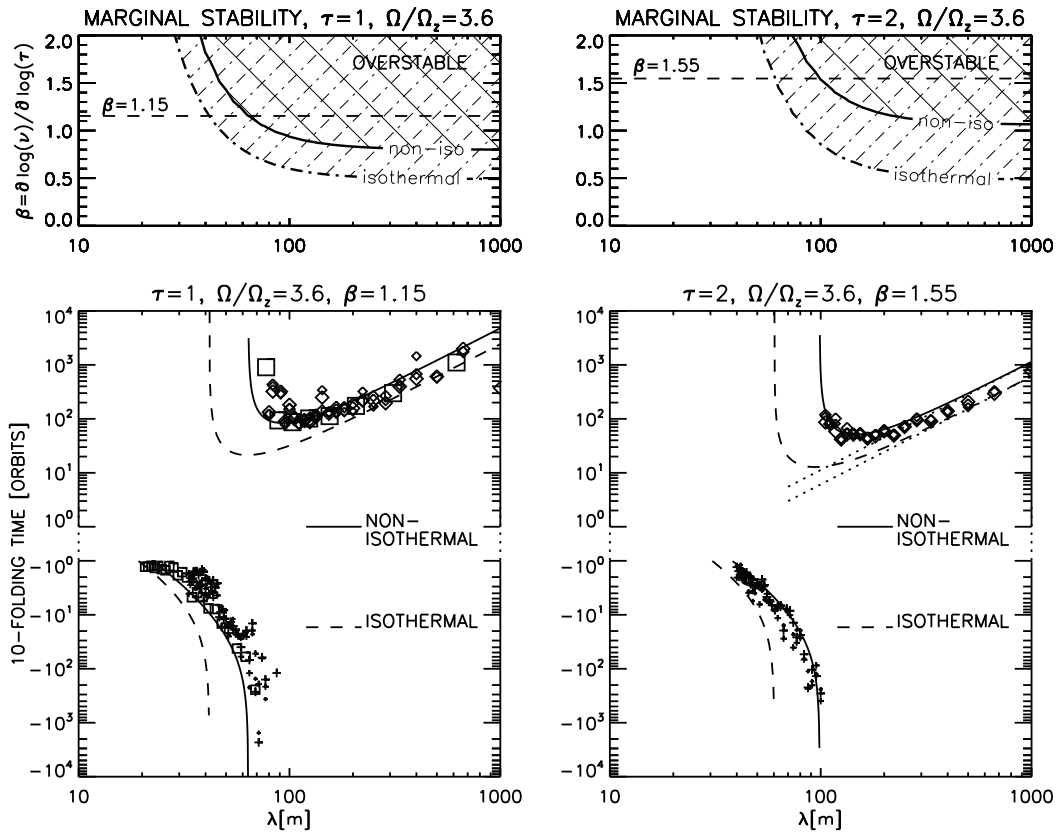

Fig. 8 Comparison of theoretical growth times to those from direct simulations. See the text for explanation. (Firgure taken from [35])

direction and the mean orbital motion points to $y$. Middle column: Side-views of the same runs. Density enhancements of the overstable oscillations (parallel to the $y$-axis) go together with a vertical splashing of the ring material. Right column: The radial velocity perturbation $u$ is shown covering the range $-0.8 \mathrm{~cm} \mathrm{~s}^{-1}<u<0.8 \mathrm{~cm} \mathrm{~s}^{-1}$. Depending on the internal particle density wakes or overstability become dominant. For the densest particles (upper row of Fig. 9) Fourier analysis of the surface density field reveals a small but hardly visible amplitude of axisymmetric overstable modes.

In Saturn's dense main rings both types of structure, oscillations due to overstability and gravitational wakes, are expected to appear simultaneously. They might offer an explanation for the small scale structure found in the spacecraft images [27] and the density profiles obtained from occultation experiments [14].

\section{Conclusions}

One of the major problems in the understanding of the behavior of granular matter is the derivation of a set of universal macroscopic equations. Difficulties arise from the lack of a separation of the scales involved: the interaction length, which is of the order of the granule size $D$, the mean free path $\langle l\rangle$ and those spatial scales relevant for changes of the state variables like density, velocities, and granular temperature. While in molecular gases these scales differ by orders of magnitude, they may be comparable for granular matter. Although kinetic 


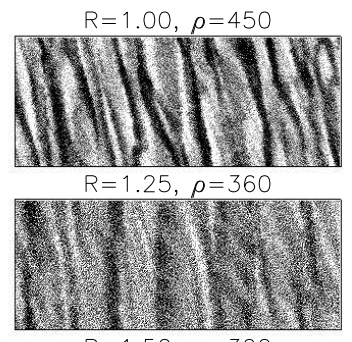

$\mathrm{R}=1.50, \rho=300$

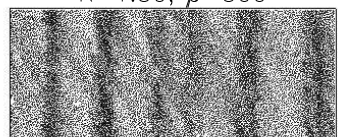

$\mathrm{R}=2.00, \rho=225$
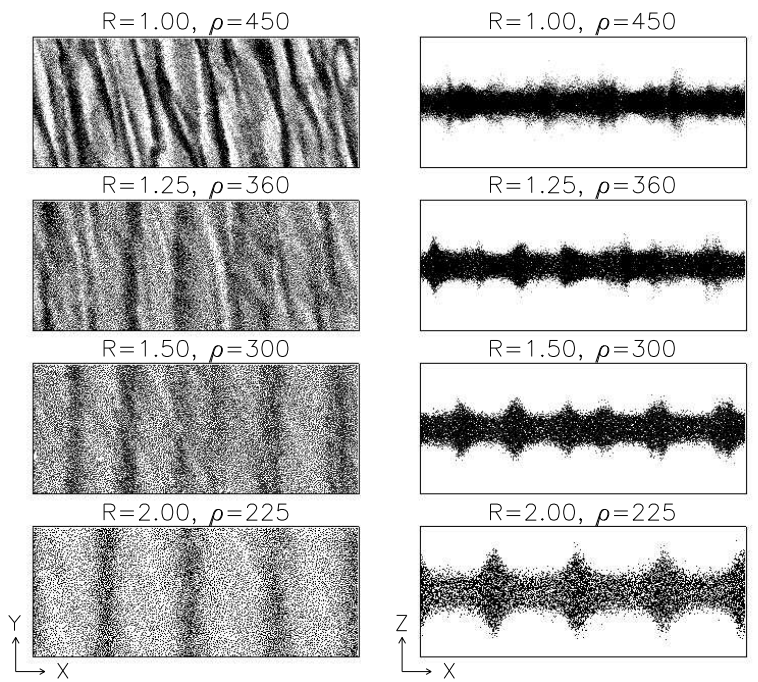

$\mathrm{R}=1.25, \rho=360$
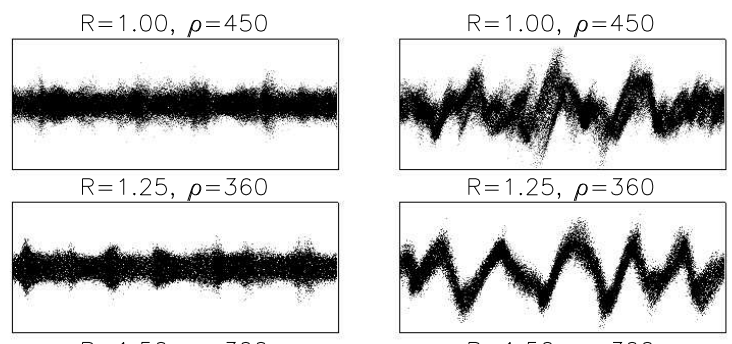

$\mathrm{R}=1.50, \rho=300$
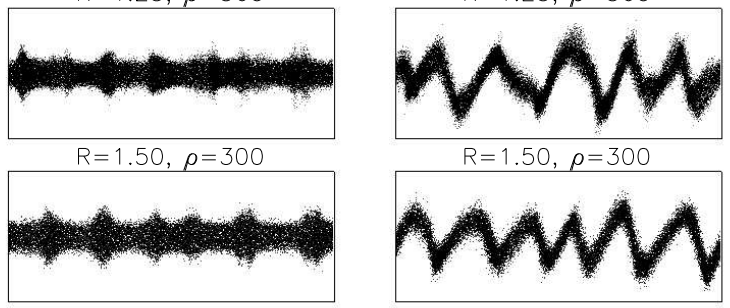

$\mathrm{R}=2.00, \rho=225$

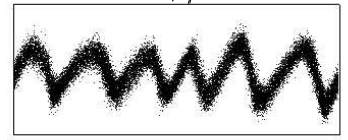

$\mathrm{R}=2.00, \rho=225$
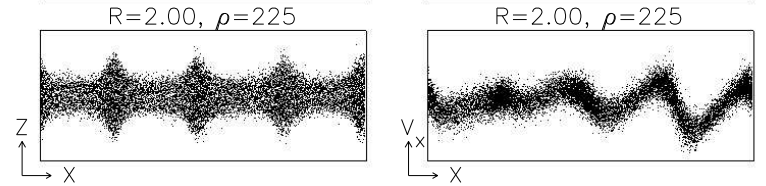

Fig. 9 Snapshots of ring structure from self-gravitating simulations. See text for explanation, (figure taken from [33]).

theory can be formulated for granular gases and the corresponding moment equations can strictly be derived, problems arise in the formulation of the necessary closure of the system - the constitutive relations. Constitutive relations have to be re-derived for a given physical situation. For example, the transport coefficients for force-free granular matter $[22,26]$ differ substantially from those of Keplerian disks.

We have demonstrated a successful application of hydrodynamics, modeling the oscillatory instability in dense planetary rings - the so-called viscous overstability. The requirement for this success is a careful analytical and numerical study of the transport processes in a dense planetary ring. With this preparation we obtained good agreement between the theoretical stability analysis, based on the hydrodynamic equations (17)-(19) and (23)-(24), and direct particle simulations. This result cannot be applied directly to proto-planetary disks, however, a careful re-derivation of transport coefficients must be carried out before.

Our studies suggest that viscous effects may play a role in the formation of small scale structures in Saturn's rings. A unique chance to detect these structures is offered by the Cassini mission. For a search of instabilities three of the 12 different sub-systems aboard the spacecraft are suitable: The imaging-sub-system (ISS), and the occultation experiments carried out with ultra-violet-spectrometer (UVIS) and the radio gain antenna (RSS). Especially the UVIS-occultations should provide the density profile of the rings at a radial resolution of about 10 meters only. This provides a solid statistical base to search for oscillations in the range 100 to 200 meters. Nevertheless, the requirements of the theoretical predictions pose a challenge to the analysis of the Cassini data. 
Table 1 Notation

\begin{tabular}{|c|c|}
\hline Symbol & Definition \\
\hline$t, \theta$ & Usual time \& slow time scale \\
\hline$\vec{r}, \vec{v}$ & Spatial vector, particle velocity \\
\hline$\nabla$ & Nabla operator \\
\hline$D, m, \bar{\rho}$ & Particle diameter, particle mass, particle mass density \\
\hline$\Omega, \Omega_{z}$ & Keplerian frequency, vertical frequency \\
\hline$f, f_{0}$ & One-particle distribution function, Maxwellian \\
\hline$f^{(2)}, Y_{E}$ & two-particle distribution function, Enskog factor \\
\hline$\vec{g}=\vec{v}_{2}-\vec{v}_{1}$ & Relative velocity between particles 1 \& 2 \\
\hline$\epsilon$ & Coefficient of restitution \\
\hline$\vec{\mu}, \vec{\Gamma}$ & One-particle phase space, Gamma space of N particles \\
\hline $\overrightarrow{\mathcal{F}}$ & External forces \\
\hline$G, V$ & Gravitational constant \& potential \\
\hline$M_{P}, M_{S}$ & Masses of the planet and satellites \\
\hline$\vec{k}$ & Unit vector between the particle centers \\
\hline$\langle l\rangle, l_{c}$ & Mean free path, characteristic length \\
\hline$c, \omega_{c}$ & Velocity dispersion, collision frequency \\
\hline$\Phi$ & Particle quantity (e.g. $m$ or $\vec{v}$ or $\vec{v}-\vec{u}$ ) \\
\hline$\langle n \Phi\rangle$ & Average of $\Phi$ with respect to $\vec{v}$ \\
\hline$n, n_{s}, \rho$ & Particle number density, surface number density, mass density \\
\hline$\vec{u}, \varepsilon$ & Mean velocity, shear tensor \\
\hline $\overrightarrow{\mathbf{p}}$ & Momentum density \\
\hline $\mathbf{P}, p$ & Stress tensor, isotropic pressure \\
\hline$\vec{Q}, T$ & Heat flux, granular temperature \\
\hline$\nu, \eta$ & Kinematic and dynamic shear viscosity \\
\hline$\zeta, \kappa, \gamma$ & Bulk viscosity, heat conductivity, granular cooling rate \\
\hline$x, y, z$ & Cartesian coordinates of a co-rotating system \\
\hline$\tau, \sigma$ & Optical depth, surface mass density \\
\hline$\langle H\rangle$ & Half thickness of the ring \\
\hline$\beta=\partial(\ln \beta) / \partial(\ln \sigma)$ & Parameter (slope) of the $\nu(\sigma)$ dependence \\
\hline$s ; k, k_{x}, k_{z}$ & Growth rate; wavenumbers \\
\hline$\vec{F}, N_{i}$ & Spatial dependencies, nonlinearity of the order $i$ \\
\hline$\partial \tilde{\mathbf{F}}, \hat{M}$ & Jacobian of $\vec{F}$, Jacobian in Fourier space \\
\hline$\vec{\Psi}$ & Linear state vector \\
\hline$\delta, \vec{\Psi}_{i}$ & Order parameter, $\mathrm{i}$ 'th component of the state vector expansion \\
\hline
\end{tabular}

\section{References}

[1] S. Araki. The dynamics of particle disks. II. Effects of spin degrees of freedom. Icarus, 76:182-198, 1988.

[2] S Araki. The dynamics of particle disks III. Dense and spinning particle disks. Icarus, 90:139-171, 1991. 
[3] S. Araki and S. Tremaine. The dynamics of dense particle disks. Icarus, 65:83-109, 1986.

[4] J. Binney and S. Tremaine. Galactic Dynamics, volume 1. Princeton University Press, 1987.

[5] F.G. Bridges, A.P. Hatzes, and D.N.C. Lin. Structure, stability and evolution of Saturn's rings. Nature, 309:333-338, 1984.

[6] N.V. Brilliantov, F. Spahn, J.-M. Hertzsch, and T. Pöschel. Model for collisions in granular gases. Phys. Rev. E, 53:5382-5392, 1996.

[7] S. Chapman and T.G. Cowling. The mathematical theory of non-uniform gases. Cambridge University Press, Cambridge, 1970.

[8] J. E. Colwell and L. W. Esposito. Origins of the rings of Uranus and Neptune. I Statistics of satellite disruptions. Journal of Geophys. Research, 97:10227-10241, 1992.

[9] J. N. Cuzzi and R. H. Durisen. Bombardment of planetary rings by meteoroids - General formulation and effects of Oort Cloud projectiles. Icarus, 84:467-501, 1990.

[10] L. Dones. A Recent Cometary Origin for Saturn's Rings? Icarus, 92:194-203, 1991.

[11] R. H. Durisen. Particle erosion mechanisms and mass redistribution in Saturn's rings. Advances in Space Research, 4:13-21, 1984.

[12] R. H. Durisen, P. W. Bode, J. N. Cuzzi, S. E. Cederbloom, and B. W. Murphy. Ballistic transport in planetary ring systems due to particle erosion mechanisms. II - Theoretical models for Saturn's A- and B-ring inner edges. Icarus, 100:364-393, 1992.

[13] R. H. Durisen, N. L. Cramer, B. W. Murphy, J. N. Cuzzi, T. L. Mullikin, and S. E. Cederbloom. Ballistic transport in planetary ring systems due to particle erosion mechanisms. I - Theory, numerical methods, and illustrative examples. Icarus, 80:136-166, 1989.

[14] L. W. Esposito, M. Ocallaghan, and R. A. West. The structure of Saturn's rings - Implications from the Voyager stellar occultation. Icarus, 56:439-452, 1983.

[15] R. G. French and P. D. Nicholson. Saturn's rings ii: Paricle sizes inferred from stellar occulation data. Icarus, 145:502-523, 2000.

[16] C. K. Goertz and G. Morfill. A new instability of Saturn's rings. ic, 74:325-330, 1988.

[17] C. K. Goertz, G. E. Morfill, W. H. Ip, E. Grün, and O. Havnes. Electromagnetic angular momentum transport in Saturn's rings. Nature, 320:141-143, 1986.

[18] P. Goldreich and S. Tremaine. The velocity dispersion in Saturn's rings. ic, 34:227-239, 1978.

[19] P. K. Haff. Grain flow as a fluid-mechanical phenomenon. J. Fluid Mech., 134:401-430, 1983. 
[20] K. H. Hämeen-Antilla and H. Salo. Generalized Theory of Impacts in Particulate Systems. Earth, Moon, and Planets, 62:47-84, 1993.

[21] H. Hwang and K. Hutter. A new kinetic model for rapid granular flow. Continuum Mech. Thermodyn., 7:357-384, 1995.

[22] J.T. Jenkins and M.T. Richman. Grad's 13-moment system for a dense gas of inelastic spheres. arma, 87:355-377, 1985.

[23] W. H. Julian and A. Toomre. Non-axisymmetric responses of differentially rotating disks of stars. Astrophys. J., 146:810-830, 1966.

[24] D. N. C. Lin and P. Bodenheimer. On the stability of Saturn's rings. Astrophys. J., 248:L83-L86, 1981.

[25] J. Lukkari. Collisional amplification of density fluctuations in Saturn's rings. Nature, 292:433-435, 1981.

[26] C.K.K. Lun, S.B. Savage, D. J. Jeffrey, and N. Chepurniy. Kinetic theories for granular flow: inelastic particles in Cuette flow and slightly inelastic particles in a general flow field. J. Fluid Mech., 140:223-256, 1984.

[27] C. C. Porco, E. Baker, J. Barbara, K. Beurle, A. Brahic, J. A. Burns, S. Charnoz, N. Cooper, D. D. Dawson, A. D. Del Genio, T. Denk, L. Dones, U. Dyudina, M. W. Evans, B. Giese, K. Grazier, P. Helfenstein, A. P. Ingersoll, R. A. Jacobson, T. V. Johnson, A. McEwen, C. D. Murray, G. Neukum, W. M. Owen, J. Perry, T. Roatsch, J. Spitale, S. Squyres, P. Thomas, M. Tiscareno, E. Turtle, A. R. Vasavada, J. Veverka, R. Wagner, and R. West. Cassini Imaging Science: Initial Results on Saturn's Rings and Small Satellites. Science, 307:1226-1236, February 2005.

[28] P. Résibois and M. De Leener. Classical kinetic theory of fluids. Wiley \& Sons, New York, 1977.

[29] H. Salo. Numerical simulations of dense collisional systems. Icarus, 90:254-270, 1991.

[30] H. Salo. Gravitational wakes in Saturn's rings. Nature, 359:619-621, 1992.

[31] H. Salo. Numerical simulations of dense collisional systems: II. Extended distribution of particle size. Icarus, 96:85-106, 1992.

[32] H. Salo. Simulations of dense planetary rings. III. Self-gravitating identical particles. Icarus, 117:287-312, 1995.

[33] H. Salo, J. Schmidt, and Spahn F. Viscous overstability in Saturn's b ring: I. Direct simulations and mesurement of transport coefficients. Icarus, 153:295-315, 2001.

[34] J. Schmidt and H. Salo. Weakly Nonlinear Model for Oscillatory Instability in Saturn's Dense Rings. Physical Rev. Letters, 90:061102(1)-(4), 2003.

[35] J. Schmidt, H. Salo, O. Petzschmann, and F. Spahn. Viscous overstability in Saturn's B-ring: II. Hydrodynamic theory and comparison to simulations . Icarus, 153:316-331, 2001. 
[36] U. Schmit and W.M. Tscharnuter. A fluid dynamical treatment of the common action of self-gravitation, collisions, and rotation in Saturn's B-ring. Icarus, 115:304-319, 1995.

[37] U. Schmit and W.M. Tscharnuter. On the formation of the fine-scale structure in saturn's B ring. Icarus, 138:173-187, 1999.

[38] Lin-Hua Shan and C. K. Goertz. On the radial structure of Saturn's B ring. Astrophys. J., 367:350-360, 1991.

[39] I.G. Shukhman. Collisional dynamics of particles in Saturn's rings. Sov. Astron., AJ 28:574-585, 1984.

[40] F. Spahn, N. Albers, M. Sremčević, and C. Thornton. Kinetic description of coagulation and fragmentation in dilute granular particle ensembles. Europhysics Letters, 67:545551, August 2004.

[41] F. Spahn, J.-M. Hertzsch, and N.V. Brilliantov. The role of particle collisions for the dynamics in planetary rings. Chaos, Solitons and Fractals, 5:1945-1964, 1995.

[42] K.D. Supulver, F.G. Bridges, S. Tiscareno, J. Lievore, and D.N.C. Lin. The sticking properties of water frost produced under various ambient conditions. Icarus, 129:538$554,1997$.

[43] S. Tremaine. On the Origin of Irregular Structure in Saturn's Rings. Astronomical Journal, 125:894-901, February 2003.

[44] J. Trulsen. Numerical simulation of jetstreams, 1:the three-dimensional case. Astrophysics and space science, 17:241-262, 1972.

[45] W. R. Ward. On the radial structure of Saturn's rings. Geophys. Res. Lett., 8:641-643, 1981.

[46] J. Wisdom and S. Tremaine. Local simulations of planetary rings. Astronomical Journal, 95:925-940, 1988. 